\title{
Dynamique de dégradation des forêts galeries et comblement du cours d'eau Agbado dans le département des Collines au Bénin
}

\author{
Luc Ollivier SINTONDJI ", Euloge K. AGBOSSOU et Boniface DEGNISSODE \\ Laboratoire d'Hydraulique et de Maîtrise de l'Eau (LHME) / Faculté des Sciences Agronomiques, \\ Université d'Abomey-Calavi (FSA /UAC). 01 BP 526 R.P. Cotonou, Benin. \\ *Auteur correspondant, E-mail: o_sintondji@yahoo.fr; agbossou.euloge@yahoo.fr \\ degnissode.forum@gmail.com ; Tél: (229) 97195578
}

\section{RESUME}

La présente étude évalue l'impact de la dégradation des galeries forestières sur le comblement de la rivière Agbado dans le département des Collines au Bénin. Elle a conduit à la description succincte des changements d'occupation des terres pendant la période d'étude et a révélé une tendance à la hausse des espaces anthropisés au détriment des zones forestières. Ainsi, les galeries forestières ont connu un taux de régression annuel de $0,87 \%$ et de conversion de $82,44 \%$. La quantité de charges solides, le poids spécifique de la vase, la superficie moyenne des sous-bassins de très faible pente, le taux de dénudation et l'indice d'agressivité climatique ont permis d'estimer la vitesse de comblement. L'indice d'agressivité climatique varie entre 25 et 150 et rend compte de la forte capacité érosive du climat dans le bassin. Les sédiments en suspension mesurés pendant la période d'août à septembre 2011 varient de $0,05 \mathrm{~g} / \mathrm{l}$ à $0,26 \mathrm{~g} / \mathrm{l}$ et permettent d'affirmer que l'érosion hydrique est l'un des facteurs responsables des matières en suspension dans le cours d'eau. Le taux de dénudation annuel calculé à partir du flux volumétrique est de $9,9 \mathrm{t} / \mathrm{km}^{2} /$ an occasionnant un comblement de $1,1 \mathrm{~mm} / \mathrm{an}$ du lit du cours d'eau.

(C) 2013 International Formulae Group. All rights reserved.

Mots clés : Bassin versant, érosion, sédimentation, ressources en eau.

\section{INTRODUCTION}

L'évaluation des écosystèmes pour le Millénaire, publiée en mars 2005, a montré que $60 \%$ des éléments d'écosystème favorables à la vie sur terre, tels que l'eau douce, l'air pur et un climat relativement stable, sont dégradés ou utilisés de manière non durable (UN, 2005). Selon le Rapport mondial des Nations Unies sur l'eau de 2006 (UN, 2006), 1,1 milliard de personnes, soit $20 \%$ de la population mondiale, n'a pas accès à une eau potable, et $40 \%$ ne disposent pas de services d'assainissement. La question de la disponibilité et de l'accès à l'eau est sans doute l'un des problèmes majeurs auquel devra faire face l'humanité durant le siècle à venir.

Le Ministère de l'Energie et de l'Eau (2009), à travers le document de la politique nationale de l'eau, a présenté le Bénin comme un pays disposant des ressources en eau relativement importantes. Mais l'apparition et la persistance, ces dernières années, de quelques indices de dégradation de la qualité de ces ressources naturelles, amène à nuancer cette appréciation. Dans ce contexte, il peut être utile de rappeler que la mesure 
quantitative et qualitative des éléments du cycle hydrologique et la mesure des autres caractéristiques de l'environnement qui influent sur l'eau constituent une base essentielle pour une gestion efficace de l'eau.

Le Bénin dispose de 2315000 ha de forêts soit $21,3 \%$ du territoire dont 114000 ha de plantations forestières. La superficie des forêts est répartie entre deux complexes de parcs nationaux et zones cynégétiques d'une superficie totale de 1357050 ha, le reste étant réparti entre 46 massifs forestiers et périmètres de reboisement (FAO, 2005). Disposant de $0,4 \%$ des forêts en Afrique et $0,06 \%$ des superficies des forêts dans le monde (FAO, 2005), les ressources forestières $\mathrm{du}$ Bénin sont donc maigres et doivent faire l'objet de la plus grande attention. En ce qui concerne le couvert végétal, le Bénin perd en moyenne 70000 ha de son couvert végétal par an (FAO, 2001). Selon Burt et Pinay (2005), les forêts galeries occupent généralement une petite fraction du paysage, mais elles jouent souvent un rôle disproportionné dans le contrôle des échanges d'eau, des produits chimiques et des particules de sol entre les terres environnantes et les cours d'eau. Il n'est l'ombre d'aucun doute que le mode d'utilisation des ressources forestières en général et des galeries forestières en particulier a un impact certain sur les ressources en eau dont dépendent les forêts galeries.

Cette étude vise, de façon globale, à évaluer la dynamique de dégradation des forêts galeries et son impact sur les ressources en eau de la rivière Agbado.

De façon spécifique, elle vise à analyser la dynamique spatio-temporelle intervenue dans l'occupation des terres et les tendances évolutives dans le département des collines au cours de ces 30 dernières années. Elle permettra aussi d'évaluer les pertes de terre dues à l'érosion hydrique dans le bassin versant et les matières solides en suspension dans la rivière Agbado et de calculer la vitesse moyenne annuelle de comblement de la rivière Agbado.

\section{MATERIEL ET METHODES}

Présentation du milieu d'étude

La zone d'étude est située dans le département des collines, entre les $7^{\circ} 27^{\prime}$ et $8^{\circ} 46^{\prime}$ de latitude nord et entre $1^{\circ} 39^{\prime}$ et $2^{\circ} 44^{\prime}$ de longitude est (Figure 1). Elle est à cheval sur les communes de Dassa-zoume, Glazoué, Savalou, Bantè et couvre une superficie d'environ $2703 \mathrm{~km}^{2}$, soit une proportion de près de $2,04 \%$ du territoire national.

\section{Milieu physique}

Le bassin versant de la rivière Agbado est à cheval sur le sous climat soudanoguinéen ouest caractérisé par une pluviométrie comprise entre 1200 et $1300 \mathrm{~mm}$ d'eau/an, répartie en une seule saison et le sous climat soudano-guinéen sud caractérisé par une pluviométrie variant de 1100 à $1200 \mathrm{~mm}$ d'eau/an et où on note des années à quatre saisons et des années où il n'y a que deux saisons (Awoyé, 2007). Le bassin est bien ensoleillé, bien éclairé durant toute l'année. Selon Akoègninou (2004), la moyenne annuelle d'insolation à la station synoptique de Savè, pour la période de 1956 à 2010 est de 2455,6 heures. Les températures restent élevées toute l'année mais elles ne sont jamais excessives. Les moyennes annuelles oscillent autour de 28,04 ${ }^{\circ} \mathrm{C}$ à Savalou. Ces moyennes, constamment élevées et presque homogènes pour l'ensemble de la région ne constituent pas un facteur limitant pour les activités agricoles.

La moyenne pluviométrique sur les vingt-quatre ans considérés sur la période (1987-2010) est de $1104 \mathrm{~mm}$. La hauteur maximale annuelle avoisine $1496,8 \mathrm{~mm}$ pour l'année 2003 (année humide), alors que la valeur minimale $755,2 \mathrm{~mm}$ est enregistrée pendant l'année 2005 qui fut une année remarquablement sèche. L'humidité relative moyenne annuelle à la station de Savè est de $68,2 \%$. Les minima mensuels sont observés en janvier $(49,2 \%)$ et les maxima en août $(81,6 \%)$. Un rapprochement entre les moyennes mensuelles pluviométriques et celles de l'humidité relative permet de constater que les mois pluvieux sont 
également ceux au cours desquels l'humidité relative de l'air est élevée.

Le bassin en étude présente des sols très variés, répartis en fonction des conditions topographiques et édaphiques du milieu. En effet, sur les bas-versants et dans les dépressions, se développent des sols hydromorphes liés aux excès d'eau temporaires ou permanents. Sur les versants moins pentus (inférieurs à 5\%) se développent des sols profonds sans gravillons ni cuirasse, ils sont favorables aux cultures annuelles. Enfin, sur les versants très pentus (supérieurs à 5\%) et les sommets, se développent des sols minéraux sur des substratums de roches affleurants ou sub-affleurants, peu profonds et gravillonnaires. Le modelé est une pénéplaine avec des dômes isolés appelés inselbergs et de petites chaînes dont l'une s'étend sur près de $20 \mathrm{~km}$ donnant à la commune son appellation du "pays de la chaîne des collines". Le relief culmine entre 120 et $500 \mathrm{~m}$ avec des pentes variant entre 3 et $10 \%$ dans les sites agglomérés. Le fleuve Ouémé est le principal cours d'eau et il reçoit la Beffa et le Zou à l'ouest, et l'Okpara à l'est. A ces principaux cours d'eau, il faut ajouter les grandes rivières telles que l'Agbado (109 km) et la Toumi. La rivière Agbado est un affluent du Zou. Le bassin versant du Zou couvre $8440 \mathrm{~km}^{2}$ et celui de l'Agbado, une superficie de $2703 \mathrm{~km}^{2}$ soit une proportion de $19,44 \%$ du département des collines. La rivière Agbado reçoit le Klou (49 km) couvrant une superficie de $560 \mathrm{~km}^{2}$ (FAO, 2009).

Le bassin versant de l'Agbado, draine toute une région avant de se jeter dans le Zou long de $250 \mathrm{~km}$ (Awoyé, 2007). Le paysage est constitué de plusieurs formations végétales composées par endroits de galeries forestières, de forêts denses sèches, semi-décidues, de forêts claires, savanes boisées, de savanes arbustives et saxicoles.

\section{Milieu humain}

$\mathrm{La}$ population $\mathrm{du}$ bassin est inégalement répartie d'une commune à l'autre, d'un arrondissement à l'autre. Les données du Recensement Général de la
Population et de l'Habitat (RGPH) des années 1992 et 2002 ont montré que cette population du département des collines est passée de 340.284 habitants en 1992 à 535.923 habitants en 2002 (Tableau 1), soit un taux d'accroissement annuel moyen de $4.7 \%$. Le bassin du Zou est occupé par différents peuples que sont par ordre d'arrivée, les Ifè, les Yoruba, les Cotocoli, les Peulh, les Mahi, les Fon, les Bétamaribè, les Adja, les Kabiai, etc., qui vivent en parfaite symbiose.

Ces différents peuples s'adonnent à de nombreuses activités dont l'agriculture qui est surtout pratiquée par les Ifè, les Mahi, les Fon, les Cotocoli, les Bétamaribè et les Adja, qui utilisent les Kabiais comme ouvriers agricoles saisonniers. Les Yoruba sont des commerçants tandis que les Peuhl sont des éleveurs de bovins. Il faut préciser que les Fon et les Adja, qui ont migré vers ce secteur sont surtout à la recherche de vastes terres fertiles qu'ils ne peuvent pas trouver chez eux.

\section{Méthodes d'étude}

La démarche utilisée découle d'une approche transversale qui est à la fois analytique et diachronique.

Etude de la dynamique de dégradation de la forêt galerie

Pour étudier la dynamique de dégradation de la galerie forestière, il a été mis en place une matrice de transition à partir des cartes d'occupation du sol des années 1972 et 2009. Cette matrice permet de décrire, de manière condensée, les changements d'état des cellules d'occupation des terres pendant une période d'étude (Schlaepfer, 2002). Le Tableau 2 présente le calcul d'une matrice des changements intervenus entre deux dates $t_{0}$ et $\mathrm{t}_{1}$, correspondant respectivement aux catégories i et $\mathrm{j}$ d'occupation des terres.

La case a $(i, j)$ de la matrice représente la superficie d'une catégorie i d'occupation des terres au temps $t_{0}$, convertie à une catégorie $\mathrm{j}$ au temps $t_{1}$. Les changements se font donc de la ligne i vers la colonne j. Dans le cadre de la présente étude, cette superficie a été calculée à partir de l'intersection des 
cellules de l'occupation des terres aux deux dates 1972 et 2009, à l'aide de l'algorithme "Intersect polygons" de l'extension Geoprocessing du logiciel Arcview 3.3. La superficie, provenant de l'intersection de deux catégories d'occupation des terres, a été reportée dans les cellules de la matrice. En référence au principe des automates cellulaires, nous considérons que les valeurs a $(i, j)$ sont obtenues sur la base de l'addition de toutes les cellules élémentaires qui sont passées de l'état i à l'état j. Dans l'analyse de la matrice de transition, on considère par exemple que si $\mathrm{i}$ ou $\mathrm{j}=1$ correspond aux champs et aux jachères et si $\mathrm{i}$ ou $\mathrm{j}=2$ correspond à la forêt claire et à la savane boisée, alors la cellule a $(2,1)$ représente la superficie de la forêt claire et de la savane boisée, qui a été convertie en champs et en jachères entre les dates $t_{0}$ et $t_{1}$. Les cellules a (i, i) de la diagonale correspondent à la superficie des zones stables qui n'ont pas évolué entre les dates $\mathrm{t}_{0}$ et $\mathrm{t}_{1}$. La somme $\mathrm{Eit}_{0}=$ $\Sigma \mathrm{a}(\mathrm{i}, \mathrm{j}), \mathrm{j}=1, \mathrm{n}$ de la ligne i représente la superficie totale de la catégorie i d'occupation des terres au temps $\mathrm{t}_{0}$. La somme Ejt1 $=\Sigma \mathrm{a}(\mathrm{i}$, $\mathrm{j}), \mathrm{i}=1, \mathrm{~m}$ de la colonne $\mathrm{j}$ représente la superficie totale de la catégorie $\mathrm{j}$ d'occupation des terres au temps $t_{1}$. Le facteur de conversion Ci de la catégorie i correspond à la somme des changements d'une catégorie $i$ vers d'autres catégories.

$$
\mathrm{Ci}=\frac{(S L i \perp a(i, i))}{S L i}
$$

où $S E i$ est la somme de la ligne $i$.

Les éléments qui sont en dehors de la diagonale représentent les changements d'occupation des terres. Par conséquent, ce sont ces valeurs qui expliquent les changements de flux de matière organique observés dans le paysage. De plus, l'analyse statistique de la dynamique de l'occupation des terres a été faite à partir du calcul du taux moyen annuel d'expansion spatiale. La formule de Bernier pour mesurer la croissance des agrégats macroéconomiques entre deux périodes données (Bernier, 1992) a été utilisée. La variable considérée ici est la superficie (S). Ainsi, pour S1 et S2, correspondant respectivement à la superficie d'une catégorie d'occupation des terres en 1972 et en 2009, le taux d'expansion spatiale moyen annuel $T$, est évalué à partir de la formule suivante:

$$
T=\frac{\left(\ln \mathrm{S}_{2}-\ln \mathrm{S}_{1}\right)}{t \ln \mathrm{e}} \times 100
$$

où : $\mathbf{t}$ est le nombre d'années d'évolution ;

In le logarithme népérien; e la base des logarithmes népériens $(e=2,71828)$.

Le contenu des différentes cartes de synthèse révèle deux situations :

- stabilité : s'il n'ya pas changement d'état au niveau de l'unité d'occupation de l'espace ;

- évolution: s'il y a eu changement d'état ; dans ce cas l'évolution peut être progressive (lorsqu'une formation moins dense en 1979 devient plus dense les autres années) ou régressive (lorsqu'une formation dense en 1979 devient moins dense les autres années).

\section{Evaluation des pertes de terre due à l'érosion hydrique}

Pour l'évaluation des pertes de terre selon le couvert végétal, des travaux de modélisation à partir du modèle SWAT (soil and water assessment tool) (Bossa, 2007) ont été réalisés. En effet, le bassin de la rivière Agbado est un sous-bassin du bassin du Zou précédemment investigué par cet auteur. La version modifiée de l'équation universelle des pertes de terre de Wischmeier a été utilisée par SWAT.

\section{Etude du comblement de la rivière Agbado}

La détermination de la vitesse de comblement de la rivière nécessite l'estimation de l'indice d'agressivité pluviométrique, du taux de dénudation et la quantification des débits solides.

\section{Estimation de l'indice d'agressivité des pluies sur le bassin-versant}

Comme nous ne disposons que des données pluviométriques journalières et mensuelles, nous avons procédé à une estimation à partir des indices d'agressivité 
climatique $(F)$ de Fournier (1962) cité par Lamouroux (1969) et Gnongbo (1996), afin de déterminer la capacité érosive du climat.

L'équation donne :

$F=\frac{p^{2}}{\mathbf{P}}$

Avec :

$p$, la pluviométrie mensuelle la plus élevée, et $\mathrm{P}$, la moyenne pluviométrique annuelle

Calcul des apports et du taux de dénudation

Calcul des apports

L'étude est basée sur les valeurs des débits liquides (Q1), donnés en $\mathrm{m}^{3} / \mathrm{s}$, mesurés à l'exutoire. Pour la mesure des sédiments en suspension, une collecte d'eau dans des bouteilles de 1,5 litre a été faite tous les deux jours à l'exutoire. Ces échantillons d'eau ont été filtrés au laboratoire avec des papiers filtres. Les matières en suspension (MES) ont été déterminées par filtration frontale des échantillons d'eau sur des filtres en ester de cellulose de porosité $0,45 \mu \mathrm{m}$. Les filtres contenant les MES ont été séchés à $105{ }^{\circ} \mathrm{C}$ à l'étuve pendant $30 \mathrm{~mm}$, puis pesés sur une balance de précision (1/10000) après passage au dessiccateur. La détermination des teneurs en MES se fait par différence de poids du filtre chargé et du filtre vierge ramenée au volume d'eau prélevé; ce qui établit la concentration, donnée en g/l. L'estimation du débit solide en suspension est liée à la mesure du débit liquide de l'écoulement par la relation :

$Q_{S}=C \cdot Q_{L}$

Avec :

$\boldsymbol{Q}_{S}$ : Débit solide en suspension $(\mathrm{kg} / \mathrm{s})$, $\boldsymbol{Q}_{L}$ : Débit liquide $\left(\mathrm{m}^{3} / \mathrm{s}\right)$ et

$C$ : Concentration ou turbidité $(\mathrm{g} / \mathrm{l})$

\section{Taux de dénudation}

Les apports solides (As) en millions de tonnes (MT), et les apports spécifiques ou taux de dénudation (Ass) en tonnes par kilomètre carré par an ( $\left.\mathrm{t} / \mathrm{km}^{2} / \mathrm{an}\right)$, sont donnés par :
As $=$ Qs.N. $10^{9}$ et Ass $=\left(\right.$ As. $\left.10^{6}\right) / \mathrm{S}$

Avec :

As : apport solide annuel, mensuel ou saisonnier (MT)

Qs : débit solide annuel, mensuel ou saisonnier $(\mathrm{Kg} / \mathrm{s})$

$\mathbf{N}$ : temps en secondes (s)

Ass : apport solide spécifique (t/ $\left./ \mathrm{km}^{2} / \mathrm{an}\right)$ et $\mathbf{S}$ : superficie du bassin versant $\left(\mathrm{km}^{2}\right)$

La détermination du taux de dénudation calculé à partir du flux volumétrique de sédiments à l'exutoire permet d'avoir une idée des pertes de terre par érosion réellement transportées dans le bassin. Ces variables permettent de quantifier, au moins approximativement à partir du débit liquide et des concentrations de matières en suspension, les charges solides et solubles transitant par le cours d'eau.

\section{Estimations de la vitesse de comblement de la rivière Agbado}

La méthode utilisée pour avoir une idée globale du comblement annuel du cours d'eau de la rivière Agbado est celle présentée par Rolf en 1992 et utilisée par Amoussou (2010) dans le bassin du Mono. De cette méthode, nous déduisons la hauteur moyenne de comblement ( $h m c$ en mm/an) du système fluvial par les relations :

$$
\begin{aligned}
h m c & =\frac{q m}{P_{s v} \times S i} \\
q m & =\frac{c\left[\frac{\left[\left(d \rrbracket_{1} \times S a v\right)\right.}{S a m}\right]}{d_{2}}
\end{aligned}
$$

Avec :

$q m=$ quantité de charges solides dans le système fluvial ( $\mathrm{t} / \mathrm{an}$ )

$c=$ charge solide mesurée de la rivière Agbado (t /an),

$d_{l}=$ moyenne des débits moyens journaliers du Klou à Logozohè $\left(\mathrm{m}^{3} / \mathrm{s}\right)$ 
$d_{2}=$ moyenne des débits moyens journaliers de Agbado au pont de Savalou ( $\left.\mathrm{m}^{3} / \mathrm{s}\right)$,

Sav = superficie en aval du bassin $\left(\mathrm{km}^{2}\right)$

Sam $=$ superficie en amont du bassin $\left(\mathrm{km}^{2}\right)$,

$P s v=$ poids spécifique de la vase en un temps $\mathrm{t}\left(\mathrm{t} / \mathrm{m}^{3}\right)$,

$S i=$ superficie moyenne des sous-bassins où la pente est plus faible $\left(\mathrm{km}^{2}\right)$.
Des deux relations (1) et (2), nous pouvons écrire :

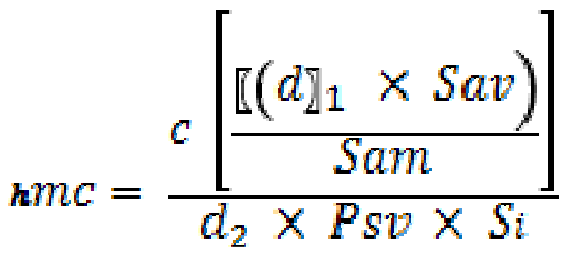

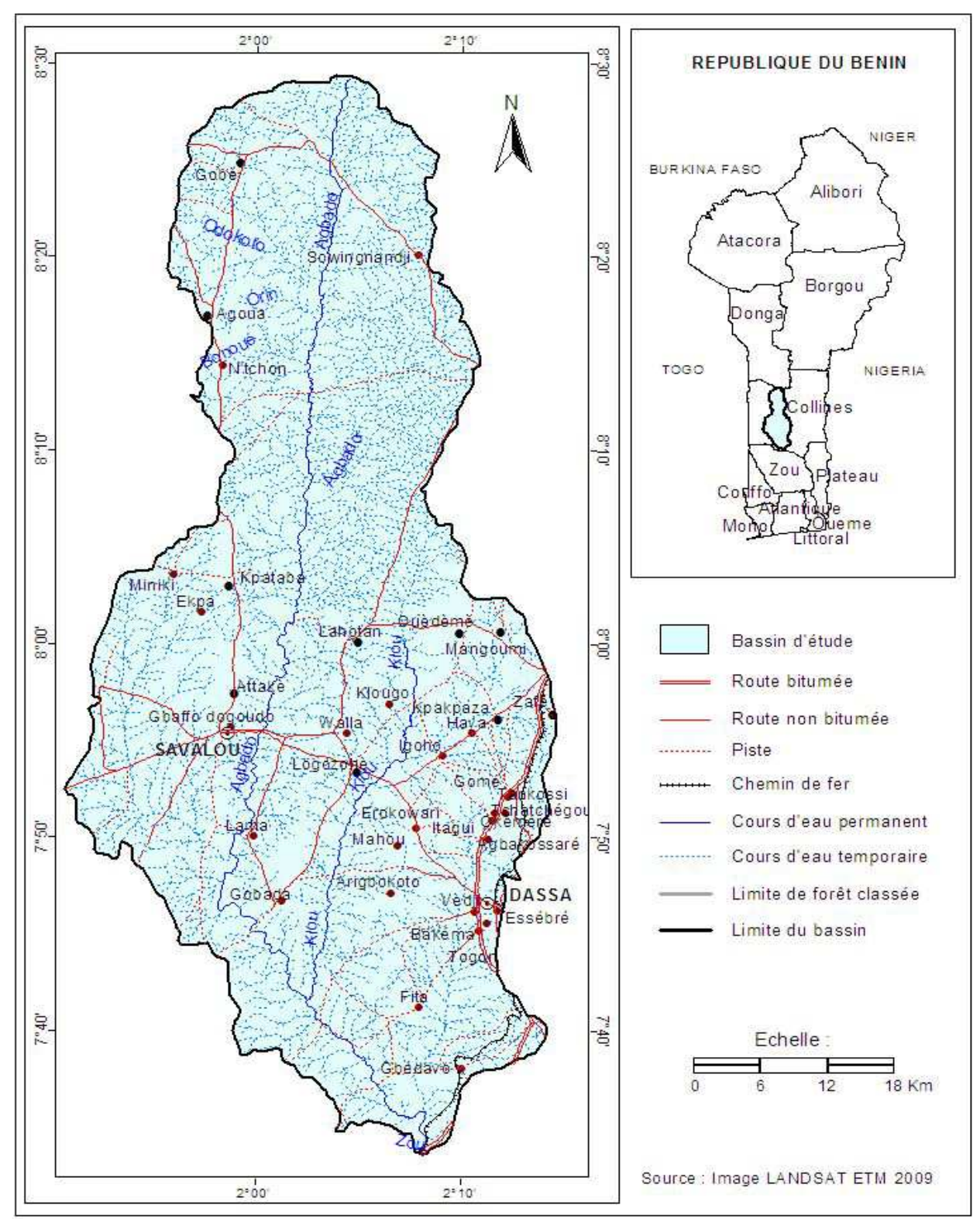

Figure 1 : Situation géographique du bassin versant de la rivière Agbado. 
Tableau 1: Population et densité par commune dans le département des Collines.

\begin{tabular}{lcccccc}
\hline \multirow{2}{*}{ Communes } & \multicolumn{2}{c}{ Population } & \multirow{2}{*}{$\begin{array}{c}\text { Superficie } \\
\left(\mathbf{k m}^{2}\right)\end{array}$} & \multicolumn{2}{c}{ Densité $\left(\mathbf{h a b} / \mathbf{k m}^{\mathbf{2}}\right)$} \\
\cline { 2 - 3 } & $\mathbf{1 9 9 2}$ & $\mathbf{2 0 0 2}$ & & & $\mathbf{1 9 9 2}$ & $\mathbf{2 0 0 2}$ \\
\hline Bantè & 46699 & 82124 & & 2675 & 17.5 & 31 \\
Dassa-zoumé & 64065 & 93967 & & 1711 & 37.4 & 55 \\
Glazoué & 59405 & 90475 & 1764 & 44.0 & 51 \\
Ouèssè & 52071 & 96850 & 2879 & 18.1 & 34 \\
Savalou & 72641 & 104749 & 2674 & 27.2 & 39 \\
Savè & 45403 & 67753 & & 2228 & 20.4 & 30 \\
Total & 340284 & 535923 & 13931 & 24.4 & 40 \\
\hline
\end{tabular}

Source : Données INSAE.

Tableau 2 : Calcul d'une matrice de transition.

\begin{tabular}{lcccc}
\hline $\begin{array}{l}\text { Catégorie } \mathbf{i} \\
\text { au temps } \mathbf{t}_{\mathbf{0}}\end{array}$ & \multicolumn{3}{c}{ Catégorie j au temps $\mathbf{t}_{\mathbf{1}}$} & $\begin{array}{c}\text { Somme Eit } \\
\text { des lignes }_{\mathbf{0}}\end{array}$ \\
\cline { 2 - 4 } & Catégorie 1 $(\mathbf{j}=\mathbf{1})$ & $\begin{array}{c}\text { Catégorie } \\
(\mathbf{j}=\mathbf{2})\end{array}$ & $\begin{array}{c}\text { Catégorie 1 } \\
(\mathbf{j}=\mathbf{3})\end{array}$ & \\
\hline Catégorie 1 $(\mathrm{i}=1)$ & $\mathrm{a}(1,1)$ & $\mathrm{a}(1,2)$ & $\mathrm{a}(1,3)$ & $\Sigma \mathrm{a}(1, \mathrm{j}), \mathrm{j}=1, \mathrm{n}$ \\
Catégorie 1 $(\mathrm{i}=2)$ & $\mathrm{a}(2,1)$ & $\mathrm{a}(2,2)$ & $\mathrm{a}(2,3)$ & $\Sigma \mathrm{a}(2, \mathrm{j}), \mathrm{j}=1, \mathrm{n}$ \\
Catégorie 1 $(\mathrm{i}=3)$ & $\mathrm{a}(3,1)$ & $\mathrm{a}(3,2)$ & $\mathrm{a}(3,3)$ & $\Sigma \mathrm{a}(3, \mathrm{j}), \mathrm{j}=1, \mathrm{n}$ \\
$\begin{array}{l}\text { Somme des Ejt } \\
\text { des colonnes }\end{array}$ & $\Sigma \mathrm{a}(\mathrm{i}, 1), \mathrm{i}=1, \mathrm{~m}$ & $\Sigma \mathrm{a}(\mathrm{i}, 2), \mathrm{i}=1, \mathrm{~m}$ & $\Sigma \mathrm{a}(\mathrm{i}, 3), \mathrm{i}=1, \mathrm{~m}$ & $\Sigma \Sigma \mathrm{a}(\mathrm{i}, \mathrm{j}), \mathrm{i}=1, \mathrm{~m}$ \\
\hline
\end{tabular}

\section{RESULTATS ET DISCUSSION}

Dynamique de l'occupation des terres

Les Figures 2 et 3 présentent les cartes d'occupation du sol du bassin versant de l'Agbado à l'exutoire de Zouto respectivement en 1972 et 2009. De ces deux figures, il ressort qu'en 1972, les agglomérations de superficie restreinte se localisaient uniquement au niveau des chefs lieu des communes et de certaines localités du bassin. Par contre les galeries forestières et les autres formations végétales couvraient de grandes superficies. Trente-sept ans plus tard, la superficie des agglomérations avait beaucoup accru, mettant en relief la dégradation du couvert végétal principalement les forêts qui ont connu une réduction drastique de leur superficie au profit des installations anthropiques.

Le Tableau 3 présente la dynamique de l'occupation des terres entre 1972 et 2009 dans le bassin de l'Agbado. L'analyse des données du Tableau 3 montre une forte emprise des activités anthropiques sur les formations naturelles. Les mosaïques de champs et jachères (MC-JA) sont passées de $520,18 \mathrm{~km}^{2}$ à $1370,55 \mathrm{~km}^{2}$ pour un taux d'expansion annuel de $0,96 \%$ et les plantations (Pl) sont passées de $7 \mathrm{~km}^{2}$ à $52,02 \mathrm{~km}^{2}$ pour un taux d'expansion de 1,99\%. Par contre les galeries forestières (FG) sont passées de 256,97 $\mathrm{km}^{2}$ à 107,63 $\mathrm{km}^{2}$, les forêts claires et les savanes boisées (FC-SB) sont passées de 345,48 à $51,96 \mathrm{~km}^{2}$ 
et les savanes à emprise agricole (SA-EA) sont passées de 1078,78 à $678,15 \mathrm{~km}^{2}$, pour des taux de régression annuels respectifs de $0,87 \% ; 1,88 \%$; et $0,46 \%$.

Mieux, l'installation de nouveaux colons agricoles et l'essor du développement agricole ont conduit à la création de hameaux de culture et à l'expansion des agglomérations existantes. Ces taux d'expansion des mosaïques de culture/jachères, des plantations et agglomérations au détriment des formations naturelles dans le bassin de l'Agbodo sont légèrement supérieurs à ceux obtenus par Tente (2002), sur les versants du massif de l'Atacora au nord-Bénin et par Oloukoï et al. (2007), dans le département des collines et se justifient par la spécificité des activités anthropiques du milieu. Cette dynamique observée dans le bassin de l'Agbado est la conséquence de la pratique de l'agriculture itinérante sur brûlis, principale technique culturale depuis des décennies dans le bassin. A cette pratique est associée l'abattage des arbres pour la fabrication du charbon et le bois de chauffe par les populations riveraines; le tout soutenu par la poussée démographique (taux annuel d'accroissement de 4,7\%, contre $3,3 \%$ pour le territoire national).

\section{L'indice d'agressivité climatique}

La Figure 4 présente l'agressivité climatique des trois principales communes du bassin. Dans la zone basse $\mathrm{du}$ bassin (Savalou), l'agressivité climatique est la plupart des années plus forte que dans la partie amont (Bantè) et peut s'expliquer par les maxima pluvieux. Cette intensité de pluie, en l'absence de couvert végétal augmente la battance des sols et occasionne un charriage important de sédiments vers le cours d'eau.

\section{Pertes en terre par couvert végétal}

Avec la légère reprise pluviométrique des années 1990, la dégradation du couvert végétal constatée sur la période 1972-2009 et l'augmentation des lames d'eau ruisselées, les pertes de terre n'ont pu que s'accentuer par rapport à la moyenne de 5,3 tonnes/ha/an trouvée pour le bassin sur la période 20012004 qui correspond à la période de calage du modèle SWAT. Mais, elles varient en fonction des types de couvert végétal et dépendent de la structure des sols dans le bassin (Amoussou et al., 2009).

\section{Taux de dénudation}

La détermination du taux de dénudation du sol (Tableau 4) calculé à partir du flux volumétrique de sédiments permet d'avoir une idée des pertes de terre par érosion transportées à travers le cours d'eau dans le bassin.

Le Tableau 5 présente outre le module (débit moyen annuel), toutes les autres données permettant l'évaluation de la vitesse de comblement de la rivière Agbado. La vitesse de comblement du cours d'eau est évaluée à $1,1 \mathrm{~mm} /$ an contre $1,6 \mathrm{~mm} / \mathrm{an}$ trouvé par Amoussou en 2010 dans le bassin du Mono, un autre bassin du sud-Bénin se trouvant à l'ouest de celui de l'Agbado. La forte agressivité climatique (plus de $50 \mathrm{~mm}$ ) dans le bassin montre l'importance des maxima pluviométriques de juin. Cette intensité de pluie, en absence de couvert végétal, occasionne un charriage des sédiments dans le plan d'eau (Amoussou et al., 2007). Dans les autres secteurs du bassin, en plus de la régression soutenue des formations végétales naturelles et de la dégradation des terres suite aux techniques agricoles traditionnelles, s'ajoutent localement les fortes pentes. Les indices d'agressivité calculés dans le bassin-versant sont inférieurs à ceux trouvés par Ezemonye et Emeribe (2012) en zone forestière au Nigéria. Balogun et al. (2012) ont trouvé des valeurs d'indice d'agressivité climatique supérieures à $160 \mathrm{~mm}$ dans l'Est du Nigéria. Ces fortes valeurs sont dues aux quantités importantes de pluie enregistrées dans les zones tropicales humides.

Ces facteurs associés aux maxima pluviométriques soutiennent à priori une forte érosivité des sols et donc un fort taux de dénudation. 5,35 tonnes/ha de sédiments sont 
transportés en moyenne par an de l'ensemble du bassin vers le réseau hydrographique selon les résultats de Bossa (2007), soit 4,51 millions de tonnes pour tout le bassin du Zou à Atchérigbé. Ces résultats se situent dans le même ordre de grandeur que ceux obtenus par Sintondji en 2005 dans le bassin de TerouIgbomakoro situé plus au nord où la perte de terre moyenne annuelle pour ledit bassin s'élève à 5 tonnes/ha alors que dans les zones de culture, elle s'élève à 16,65 tonnes/ha.

La vitesse de comblement $(1,1 \mathrm{~mm} / \mathrm{an})$ de la rivière Agbado est inquiétante car elle correspond à un dépôt de sédiments de 49.229,9 t/an, hormis les matériaux en suspension; ce qui réduit la capacité de stockage de la rivière Agbado et la qualité de la ressource en eau. Par ailleurs, il faut signaler que la majeure partie des sédiments est simplement délocalisée d'un endroit à un autre du bassin et que toutes les particules drainées ne parviennent pas à l'exutoire du bassin. Une quantité non négligeable de particules se déposent sur le fond du cours d'eau du fait de la faiblesse des pentes et occasionne son comblement.

Tableau 3: Dynamique de l'occupation des terres entre 1972 et 2009 dans le bassin de l'Agbado.

\begin{tabular}{ccccccc}
\hline $\begin{array}{c}\text { Unité de } \\
\text { végétation }\end{array}$ & $\begin{array}{c}\text { Superficie } \\
\text { en 1972 }\end{array}$ & Proportion & $\begin{array}{c}\text { Superficie } \\
\mathbf{e n ~ 2 0 0 9}\end{array}$ & Proportion & $\begin{array}{c}\text { Taux de } \\
\text { régression }\end{array}$ & $\begin{array}{c}\text { Taux de } \\
\text { progression }\end{array}$ \\
\cline { 2 - 7 } & $\left.\mathbf{k m}^{\mathbf{2}}\right)$ & $\mathbf{\%}$ & $\mathbf{\mathbf { k m } ^ { 2 } )}$ & $\mathbf{\%}$ & $\mathbf{\%}$ & $\boldsymbol{\%}$ \\
\hline FG & 256,97 & 9,45 & 107,63 & 3,98 & $-0,87$ & - \\
FC-SB & 345,48 & 12,71 & 51,96 & 1,92 & $-1,88$ & - \\
SA-A & 404,58 & 14,89 & 305,08 & 11,29 & $-0,28$ & - \\
SA-SA & 60,5 & 2,24 & 60,5 & 2,24 & - & 0,00 \\
SA-EA & 1078,78 & 39,69 & 678,15 & 25,09 & $-0,46$ & \\
MC-JA & 520,18 & 19,14 & 1370,55 & 50,70 & - & $+0,96$ \\
Pl & 7 & 0,26 & 52,02 & 1,92 & - & $+1,99$ \\
Agg & 44,78 & 1,65 & 77,13 & 2,85 & - & $+0,58$ \\
Total & 2703,02 & 100,00 & 2703,02 & 100,00 & $-3,49$ & $+3,49$
\end{tabular}

Source : Statistiques évaluées à partir des cartes d'occupation des terres de 1972 et de 2009

Légende : $(+)$ = extension, $(-)=$ régression.

Tableau 4: Taux de dénudation du sol.

\begin{tabular}{|c|c|c|c|c|c|c|}
\hline $\begin{array}{l}\text { Cours } \\
\text { d'eau }\end{array}$ & années & $\mathrm{Ql}\left(\mathrm{m}^{3} / \mathrm{s}\right)$ & $\mathrm{C}(\mathrm{g} / \mathrm{l})$ & Qs $(\mathrm{Kg} / \mathbf{s})$ & $\operatorname{As}(\mathbf{t})$ & $\begin{array}{c}\text { Ass } \\
\left(\mathbf{t} / \mathrm{km}^{2} / \mathbf{a n}\right) \\
\end{array}$ \\
\hline Agbado & 2011 & 4,49 & 0,189 & 0,850 & 26761,76 & 9,9 \\
\hline
\end{tabular}


Tableau 5: Données ayant servi à l'évaluation de la vitesse de comblement de la rivière Agbado.

\begin{tabular}{lccc}
\hline Dénomination & Symbole & Unité & Valeur \\
\hline Débit moyen annuel sur Agbado (module) & $(d l)$ & $\mathrm{m}^{3} / \mathrm{s}$ & 4,49 \\
Débit moyen annuel sur klou (module) & $(d 2)$ & $\mathrm{m}^{3} / \mathrm{s}$ & 1.72 \\
Superficie en amont du bassin & $($ Sam $)$ & $\mathrm{km}^{2}$ & 1280 \\
Superficie en aval & $($ Sav $)$ & $\mathrm{km}^{2}$ & 902 \\
Superficie des sous bassins de pente nulle & $($ Si $)$ & $\mathrm{km}^{2}$ & 521 \\
Poids spécifique de la vase & $($ Psv $)$ & $\mathrm{t} / \mathrm{m}^{3}$ & 0,09 \\
Les apports des sédiments en suspension & $(c)$ & $\mathrm{t} / \mathrm{an}$ & 26761,76 \\
dans la rivière Agbado & $q \mathrm{qm}$ & $\mathrm{t} / \mathrm{an}$ & 49229,9 \\
Quantité de charge solide dans le système Agbado-Klou & $\mathrm{hmc}$ & $\mathrm{mm} / \mathrm{an}$ & 1,1 \\
Vitesse moyenne annuelle de comblement & & &
\end{tabular}

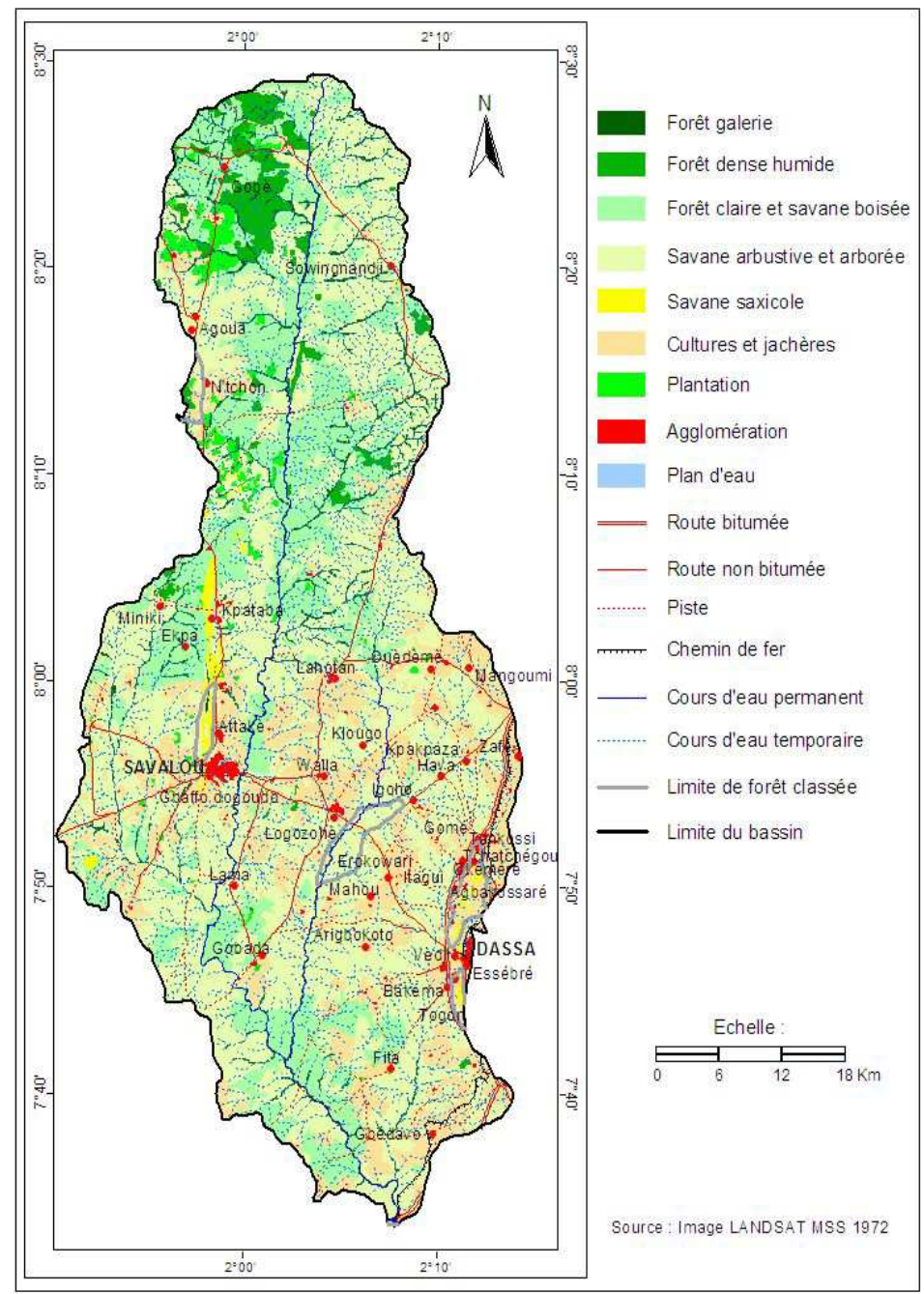

Figure 2: Occupation du sol en 1972 dans le bassin versant d'Agbado à l'exutoire de Zouto. 


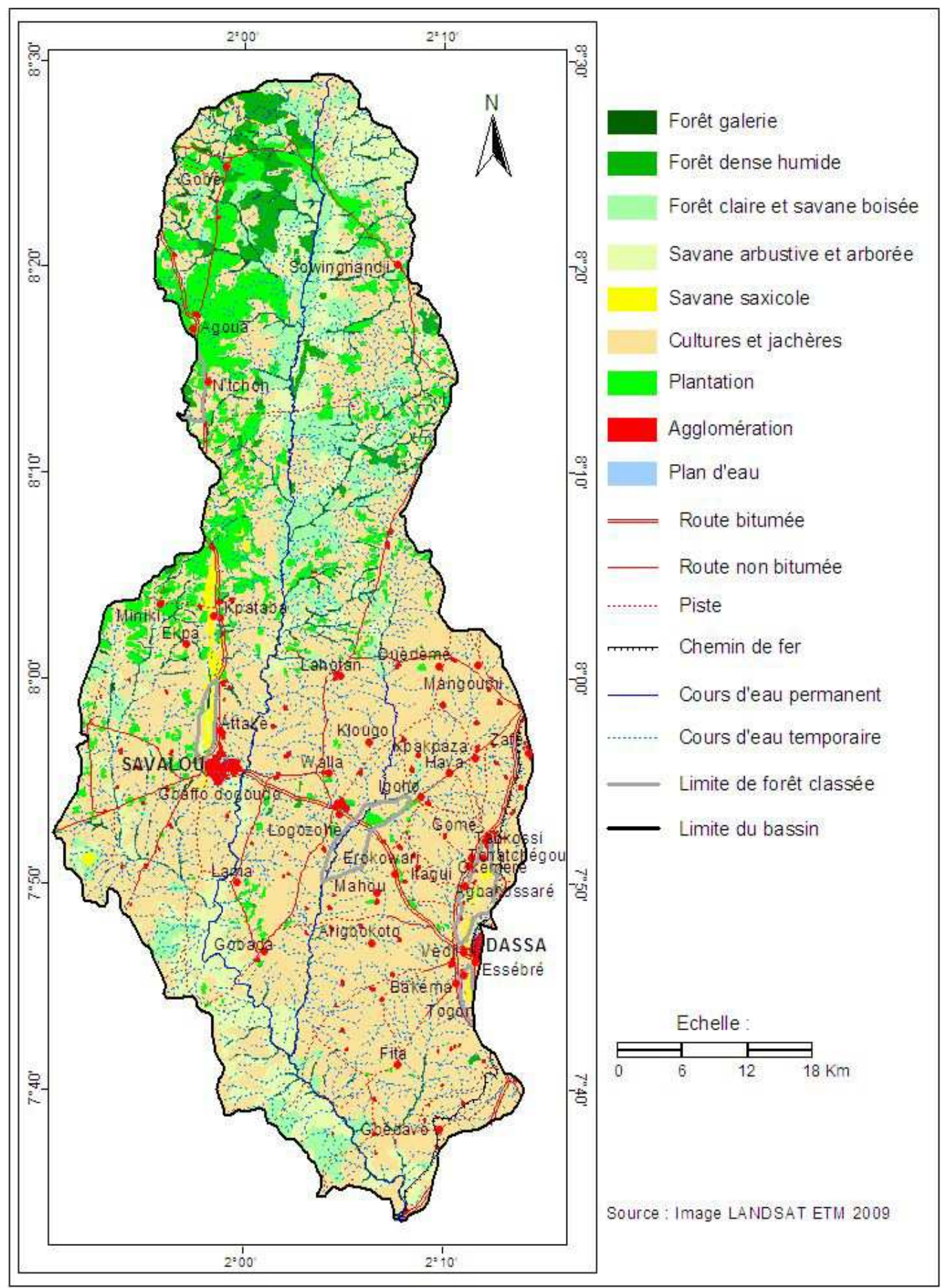

Figure 3: Occupation du sol en 2009 dans le bassin versant de Agbado à l'exutoire de Zouto. 


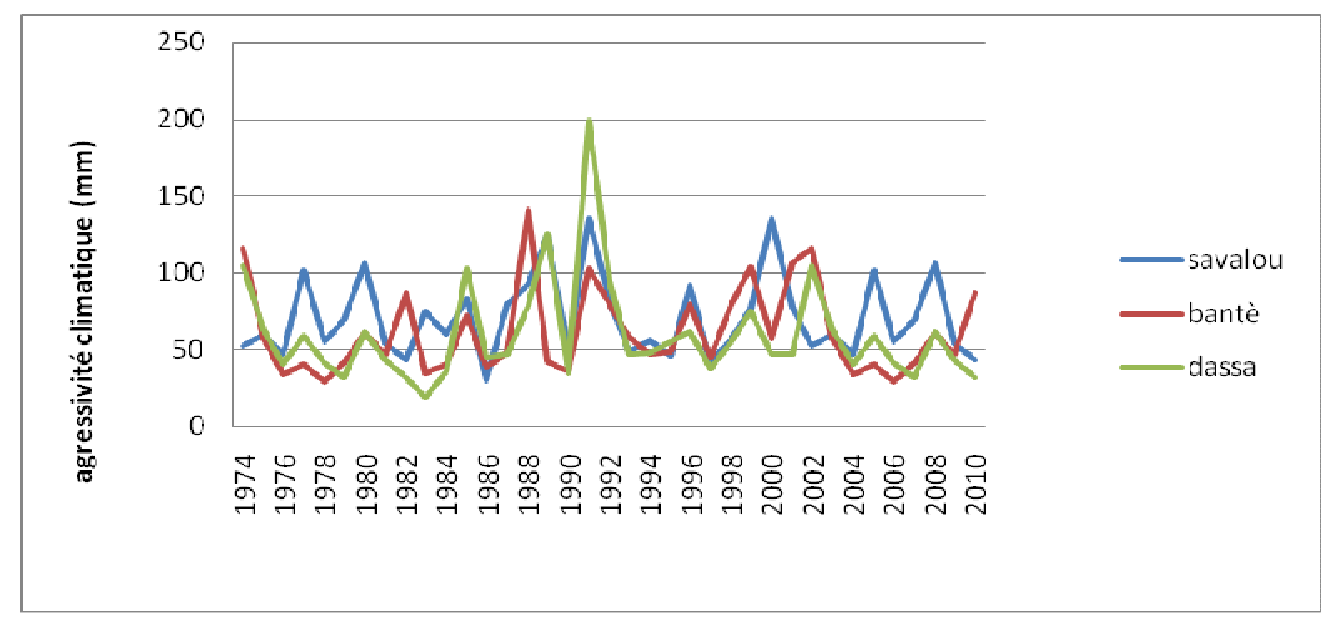

Figure 4 : Agressivité climatique des trois principales communes du bassin versant.

\section{Conclusion}

Les galeries forestières du bassin de l'Agbado ont connu un taux de régression annuel de $0,87 \%$ entre 1972 et 2009 . Cette dégradation rend vulnérables les berges du cours d'eau aux actions érosives des eaux de ruissellement et occasionne des pertes de terre évaluées à 5,3 tonnes/ha/an. Ces pertes sont plus importantes sur les terres agricoles $(17,7$ tonnes/ha/an), signe d'une forte érosion des champs situés aux flancs des collines. Tout ce déplacement de terre entraîne un comblement $\mathrm{du}$ lit du cours d'eau à raison de $1,1 \mathrm{~mm} / \mathrm{an}$. L'utilisation des terres et le couvert végétal constituent donc des paramètres clés au sein du cycle hydrologique qu'il faudra maîtriser pour une gestion durable des ressources en eau de la rivière Agbado.

\section{REMERCIEMENTS}

La présente étude a été financée par le Projet néerlandais NPT 145 du Laboratoire d'Hydraulique et de Maîtrise de l'Eau de la Faculté des Sciences Agronomiques (FSA) de l'Université d'Abomey-Calavi (UAC). Nous témoignons ici toute notre gratitude aux responsables dudit projet.

\section{REFERENCES}

Akoègninou A. 2004. De l'étude des formations végétales des sites constitutifs de réserves biologiques dans les zones humides du Sud Bénin. MAB/CBRST, 22. Amoussou E, Oyédé LM, Boko M. 2007. Variabilité pluviométrique et flux de turbidité dans le complexe Chenal Ahôlac Ahémé au Bénin (Afrique de l'Ouest). Actes du XX ème colloque AIC. In Climat, Tourisme et Environnement de Carthage. Tunisie ; 81-86.

Amoussou E, Camberlin P, Boko M, Perard J. 2009. Impact de la variabilité climatique sur les apports Liquides dans la basse vallée du mono (Bénin, Afrique de l'ouest). Actes XXIIème colloque de l'Association Internationale de Climatologie (AIC) : «Extrêmes climatiques: genèse, modélisation et impacts ». Cluj Napoca, Romanie, numéro spécial, 35-40.

Amoussou E. 2010. Variabilité pluviométrique et dynamique hydrosédimentaire du bassin-versant du complexe fluvio-lagunaire Mono-AhéméCouffo (Afrique de l'Ouest). Thèse de 
doctorat, Université de Bourgogne CRC / CNRS - UMR 5210 Paris, p. 333.

Awoyé R. 2007. Modélisation du bilan hydrologique du bassin versant du Klou : contribution à la gestion durable des ressources en eau dans le Zou. Thèse d'ingénieur FSA/UAC, Bénin, p. 100.

Balogun I, Adegun O, Adeaga O. 2012. An Assessment of Rainfall Erosivity in Parts of Eastern Nigeria: A Case Study of Owerri and Enugu. Hydrology for Disaster Management. Special Publication of the Nigerian Association of Hydrological Sciences. 2012: 259-265.

Bernier B. 1992. Introduction à la Macroéconomie. Dunod : Paris ; p. 217.

Bossa A. 2007. Modélisation du bilan hydrologique dans le bassin du Zou à l'exutoire d'Atchérigbé: Contribution à l'utilisation durable des ressources en eau. Mémoire de DESS FSA/UAC, Bénin, p. 111.

Burt TP, Pinay, G. 2005. Linking Hydrology and Biogeochemistry in Complex Landscapes. Progress in Physical Geography, 29(3): 297-316.

Ezemonye MN, Emeribe CN. 2012. Rainfall erosivity in Southeastern Nigeria. Ethiopian Journal of Environmental Studies and Management (EJESM), 5(2): 112-122.

FAO. 2001. Situation des forêts du monde. FAO, Rome, p.181.

FAO. 2005. Situation des forêts du monde. Rome, p. 123.

FAO. 2009. Situation des forêts du monde. Rome, p. 152.

Fournier F. 1962. Mesure de l'Erosion, Principes de Base et Incidence de l'Instrumentation Nouvelle sur L'Etablissement des Réseaux de Mesure. Bureau Interafricain des sols, 57 rue Cuvier, Paris: France ; 343-356.
Gnongbo TY. 1996. Le Togo méridional: étude de géographie physique. Thèse de Doctorat Université de Bordeaux III, opt. Géo. phys. tropic., p. 306.

Lamouroux M. 1969. Notice Explicative $N^{\circ} 34$ de la Carte Pédologique du Togo au 1/1 000 000. ORSTOM: Paris; p. 91.

Ministère de l'Energie et de l'Eau. 2009. Politique Nationale de l'Eau. MEE: Cotonou ; P.73.

Oloukoi J, Mama V, Agbo FB. 2007. Modélisation de la dynamique de l'occupation des terres dans le département des collines au bénin. Télédétection, 6(4): 305-323.

Schlaepfer R. 2002. Analyse de la dynamique du paysage. Laboratoire de gestion des écosystèmes (GECOS), École polytechnique fédérale de Lausanne, Lausanne, p.11.

Sintondji L. 2005. Modeling the rainfallrunoff process in the Upper Ouémé catchment (Terou in Benin republic) in a context of global change: extrapolation from the local to the regional scale. $\mathrm{PhD}$ Thesis. Mathematics and Natural Sciences Faculty /University of Bonn, Germany, p. 161.

Tente B. 2002. Dynamique actuelle de l'occupation du sol dans le massif de l'Atacora : secteur Perma-Toucountouna. Mémoire de DEA, École doctorale pluridisciplinaire, Université nationale du Bénin, p.83.

United Nations (UN). 2005. Objectifs du Millénaire pour le développement, Département de l'information de l'ONU, DPI/2390 - mai 2005 - 35M DDDD.

United Nations (UN). 2006. Rapport mondial des Nations Unies sur l'eau. 9-iii-2006, p. 524. 